\title{
The CD36 Ligand-Promoted Autophagy Protects Retinal Pigment Epithelial Cells from Oxidative Stress
}

\author{
Marie-France Dorion (D), ${ }^{1,2}$ Mukandila Mulumba $\left(\mathbb{D},{ }^{2}\right.$ Shuya Kasai $\mathbb{D}^{3},{ }^{3}$ Ken Itoh $\left(\mathbb{D},{ }^{3}\right.$ \\ William D. Lubell $\mathbb{D}^{4}{ }^{4}$ and Huy Ong $\mathbb{D}^{2}$ \\ ${ }^{1}$ Department of Neurology and Neurosurgery, Montreal Neurological Institute, McGill University, 3801 University Street, Montreal, \\ QC, Canada H3A $2 B 4$ \\ ${ }^{2}$ Faculty of Pharmacy, Université de Montréal, 2940 Chemin de Polytechnique, Montreal, QC, Canada H3T 1J4 \\ ${ }^{3}$ Department of Stress Response Science, Center for Advanced Medical Research, Hirosaki University Graduate School of Medicine, \\ 5 Zaifu-cho, Hirosaki 036-8562, Japan \\ ${ }^{4}$ Department of Chemistry, Université de Montréal, 2900 Boulevard Édouard Montpetit, Montreal, QC, Canada H3T 1J4
}

Correspondence should be addressed to Huy Ong; huy.ong@umontreal.ca

Received 20 November 2020; Revised 27 February 2021; Accepted 17 March 2021; Published 28 March 2021

Academic Editor: Stefano Falone

Copyright (C) 2021 Marie-France Dorion et al. This is an open access article distributed under the Creative Commons Attribution License, which permits unrestricted use, distribution, and reproduction in any medium, provided the original work is properly cited.

\begin{abstract}
The retinal pigment epithelium (RPE) performs many functions that maintain photoreceptor health. Oxidative damage to the RPE is a critical component in the pathogenesis of eye diseases such as age-related macular degeneration (AMD). Ligands of the cluster of differentiation 36 (CD36) have previously preserved photoreceptor integrity in mouse models of AMD. The cytoprotective effect of the CD36 ligand MPE-001 on RPE cells has now been elucidated employing a model of oxidative stress. Sodium iodate $\left(\mathrm{NaIO}_{3}\right)$ induced formation of reactive oxygen species and apoptosis in human RPE cells, which were decreased by MPE-001 without affecting antioxidant enzyme transcription. Immunoblotting and immunostaining assays showed a restorative effect of MPE-001 on the autophagic flux disrupted by $\mathrm{NaIO}_{3}$, which was associated with an increase in syntaxin 17-positive mature autophagosomes. The cytoprotective effect of MPE-001 was completely abolished by the autophagy inhibitors wortmannin and bafilomycin A1. In conclusion, we report for the first time an autophagy-dependent protection of RPE cells from oxidative stress by a CD36 ligand.
\end{abstract}

\section{Introduction}

The retinal pigment epithelium (RPE) is a monolayer of polarized epithelial cells located posterior to the neuroretina in close contact with photoreceptors. Responsible for the maintenance of photoreceptors, the RPE performs daily phagocytosis of shed photoreceptor outer segments (POS). High metabolic activity combined with exposure to light and photochemical reactions in the oxygenated environment render the RPE prone to oxidative stress. Oxidative damage to the RPE can lead to dysfunction with cell loss, inflammation, and degeneration of photoreceptors, characteristics of age-related macular degeneration (AMD) [1].
AMD is a leading cause of visual impairment in the elderly population [2] and is categorized into wet (neovascular) and dry (atrophic, nonneovascular) forms with distinct pathophysiological features. Hallmarks of dry AMD include RPE abnormality with accumulation of a complex deposit of lipids and proteins called drusen and atrophy of the photoreceptor layer in the macular region of the retina [1]. Although therapy is available for the wet form, no cure nor treatment exists to prevent dry AMD [3]. The role of oxidative stress in the pathogenesis of AMD is supported by observations of an AMD-like phenotype in mice with a genetic deficiency in either superoxide dismutase 1 or 2 (Sod1 [4] or Sod2 [5]) or nuclear factor erythroid 2-like 2 ( Nfe2l2) 
[6]. Furthermore, smoking tabacco is a risk factor of AMD [7]. Increased expression of antioxidant enzymes [8] and mitochondrial DNA damage [9] have been observed in the eyes of AMD patients.

Autophagy is an adaptive mechanism for recycling intracellular components to protect cells against oxidative damage. During autophagy, specialized double-membrane vesicles called autophagosomes form, targeting and engulfing damaged proteins and organelles for lysosomal degradation. After autophagy initiation, the nascent double-membrane phagophores elongate to sequestrate cargo and close to form mature autophagosomes, which subsequently fuse with lysosomes [10]. Autophagy malfunction has been associated with the pathogenesis of AMD. Decreased autophagic degradation activity, the so-called autophagic flux, and increased susceptibility to oxidative damage have been observed in the RPE cells of AMD patients [11]. Moreover, RPE-specific deficiency of autophagy-related 5 or 7 (Atg5 or Atg7) genes has been shown to lead to retinal degeneration in mice [12].

The scavenger receptor cluster of differentiation 36 (CD36) is well known as a transporter of fatty acids into cells [13]. Playing a role in the innate immune response and lipid homeostasis, CD36 is expressed in diverse cellular types including RPE cells [14]. Endogenous ligands of CD36, several of which promote inflammation, are known to accumulate within the retina in $\mathrm{AMD}$, including $\beta$-amyloids [15], oxidized low-density lipoproteins [16], and advanced glycation end products [17]. In addition, CD36 is involved in lipid handling in the diseased retina by mediating the clearance of subretinal deposits in mice [18]. The selective CD36 ligand EP80317, a derivative of growth hormone-releasing peptide6 (GHRP-6), has been shown to preserve photoreceptor function in Apoe - / - mice fed a high-fat high-cholesterol diet [18]. Furthermore, the azapeptide MPE-001 (His-D-TrpAla-azaTyr-D-Phe-Lys- $\mathrm{NH}_{2}$ ), a semicarbazide derivative of GHRP-6 that exhibits high CD36 binding affinity [19], attenuates photooxidative stress-induced subretinal inflammation in mice and prevents photoreceptor degeneration [20]. However, the effect of these unique synthetic CD36 ligands on RPE cell redox status has yet to be documented.

In the present study, we examined the potential of CD36 as a target to modulate oxidative stress in the RPE. Application of the azapeptide MPE-001 on human RPE cell lines exposed to oxidative stress has elucidated the role of autophagy in the cytoprotective mechanism of action. Oxidative stress was induced by sodium iodate $\left(\mathrm{NaIO}_{3}\right)$, an oxidant known to cause retinal degeneration through selective damage to the RPE in animals [21]. In RPE cell culture systems, $\mathrm{NaIO}_{3}$ has been used to reproducibly model oxidative stress involved in AMD development [22-28].

\section{Materials and Methods}

2.1. Antibodies and Reagents. Antibodies against HMOX-1 (5061S), GAPDH (2118S), LAMP1 (9091S), and LC3B (2775S) were purchased from Cell Signaling Technology. Antibodies against STX17 (PA5-40127) and ZO-1 (617300) were from Invitrogen. Antibodies against TOMM20 (H00009804-M01) were from Abnova. Sodium iodate was purchased from Wako Pure Chemical Industries. Bafilomycin A1, wortmannin, and $N$-acetyl-L-cysteine (NAC) were from Sigma-Aldrich. MitoTEMPO and mitoquinol (MitoQ) were from Cayman Chemical. Trifluoromethoxy carbonyl cyanide phenylhydrazone (FCCP) was from Agilent Technologies. Solutions of $\mathrm{NaIO}_{3}$ or NAC were freshly prepared in complete media before each experiment. The $\mathrm{pH}$ of NAC-containing media was adjusted back to the original $\mathrm{pH}$ using sodium hydroxide.

2.2. hTERT RPE-1 Culture. hTERT RPE-1 cells (American Type Cell Collection) were cultured in complete medium (DMEM/Ham's F12 media with 10\% FBS, $100 \mathrm{U} / \mathrm{mL}$ penicillin and $100 \mu \mathrm{g} / \mathrm{mL}$ streptomycin). Cells were maintained at $37^{\circ} \mathrm{C}$ under a $5 \% \mathrm{CO}_{2}$ atmosphere. Cells were used from passages between 8 and 18. After each passage, cells were incubated for at least $48 \mathrm{~h}$ to reach $90-100 \%$ confluence before treatments, unless otherwise specified.

2.3. Reactive Oxygen Species (ROS) Detection. $\mathrm{CM}-\mathrm{H}_{2} \mathrm{DCFDA}$ and MitoSOX Red (Invitrogen) were used to assess total cellular ROS and mitochondrial superoxide formation, respectively. Production of ROS was assessed $2 \mathrm{~h}$ following $\mathrm{NaIO}_{3}$ exposure, before the occurrence of apparent cell death. Cells in a black 96-well plate were incubated with $10 \mu \mathrm{M}$ CM$\mathrm{H}_{2}$ DCFDA for $30 \mathrm{~min}$ or with $2 \mu \mathrm{M}$ MitoSOX Red for $15 \mathrm{~min}$ in phosphate-buffered saline (PBS) at $37^{\circ} \mathrm{C}$. Fluorescence intensity (excitation/emission $=495 / 525$ for CM$\mathrm{H}_{2}$ DCFDA and 510/580 for MitoSOX Red) was measured using a Synergy H1 Hybrid Multi-Mode Microplate Reader (BioTek Instruments). All conditions were analyzed at least in triplicate.

2.4. Viability Assay. Cell Counting Kit-8 (CCK-8) (Dojindo Molecular Technologies) was used for the assessment of cellular viability. In a 96-well plate, 2500 cells per well were cultured for $48 \mathrm{~h}$, treated, and assessed for viability following the manufacturer's protocols. All conditions were analyzed at least in triplicate.

2.5. Caspase 3/7 Activity. In a black 96-well plate, cells were incubated with $5 \mu \mathrm{M}$ CellEvent ${ }^{\mathrm{TM}}$ Caspase-3/7 Green Detection Reagent (Invitrogen) in PBS with 5\% FBS for $30 \mathrm{~min}$ at $37^{\circ} \mathrm{C}$ and counterstained with Hoechst 33342 . Image acquisitions and analysis were carried out using a CQ1 confocal quantitative image cytometer to determine the proportion of cells with active caspase 3/7. All conditions were analyzed at least in triplicate.

2.6. Mitochondrial Membrane Potential. Mitochondrial depolarization was assessed using JC-1 dye (Invitrogen). In a black 96-well plate, 5000 cells per well were cultured for $48 \mathrm{~h}$, treated, and incubated with $2.5 \mu \mathrm{g} / \mathrm{mL}$ of JC- 1 dye in serum-free media for $20 \mathrm{~min}$ at $37^{\circ} \mathrm{C}$. Fluorescence intensity was measured using a Synergy H1 Hybrid Multi-Mode Microplate Reader, and the red (excitation/emission $=535 / 590)$ to green (excitation/emission $=485 / 530)$ fluorescence intensity ratio was determined. All conditions were analyzed at least in triplicate. Cells were additionally stained with Hoechst 
$33342(1 \mu \mathrm{g} / \mathrm{mL})$ for imaging purposes. Images were obtained using a ZEISS Axio Observer.

2.7. Transfection. For the RNA interference of CD36, cells were seeded overnight and then transfected with either ON-TARGETplus human CD36 siRNA SMART pool or siGENOME RISC-Free Control (Dharmacon) using INTERFERin siRNA/miRNA Transfection Reagent (final concentration of $0.15 \%$; Polyplus-transfection). All procedures were performed according to the manufacturer's recommendations. Knockdown efficiency was assessed by qRT-PCR using GAPDH and YWHAZ as endogenous controls.

For the visualization of autophagosomes and the flow cytometry assessment of autophagic flux, cells were transfected with Premo Autophagy Sensor LC3B-GFP, BacMam 2.0, and a Premo ${ }^{\mathrm{TM}}$ Autophagy Tandem Sensor RFP-GFPLC3B Kit (Invitrogen). Cells were incubated with 40 particles per cell for 48 hours before carrying out downstream assays. All procedures were performed according to the manufacturer's recommendations.

2.8. Quantitative Reverse Transcription Polymerase Chain Reaction ( $q R T-P C R)$. RNA was extracted using a RNeasy mini kit (QIAGEN) with some modifications to the recommended protocol. Briefly, cells were lysed with RiboZol RNA Extraction Reagent (VWR Life Science), mixed with one-third volume of chloroform, and centrifuged at $10000 \mathrm{~g}$ for $18 \mathrm{~min}$ at $4^{\circ} \mathrm{C}$. The aqueous phase supernatant was mixed with isopropanol and transferred to RNeasy columns. The manufacturer's protocol was followed from this step onward. The qPCR was performed using a ViiA 7 Real-Time PCR System (Life Technologies). Results were analyzed with the $2^{-\triangle \Delta C t}$ method, using YWHAZ and PPIA as endogenous controls. Primer sequences are shown in supplementary materials (Table S1).

2.9. ARPE-19 Cell Differentiation and Treatments. ARPE-19 cells (American Type Cell Collection) were cultured in complete medium and differentiated as previously described with slight modifications [29]. Briefly, cells were seeded at a density of 166000 cells $/ \mathrm{cm}^{2}$ on Matrigel- (Corning) coated Transwell 96-well inserts (Corning) and cultured for two weeks in MEM-Nic: Minimum Essential Medium alpha with L-glutamine (Wisent Bioproducts), 1\% FBS, $100 \mathrm{U} / \mathrm{mL}$ penicillin, $100 \mu \mathrm{g} / \mathrm{mL}$ streptomycin, $0.1 \mathrm{mM}$ Non Essential Amino Acids (HyClone), 1\% N1 supplement (Sigma-Aldrich), $0.25 \mathrm{mg} / \mathrm{mL}$ taurine (Sigma-Aldrich), $20 \mathrm{ng} / \mathrm{mL}$ hydrocortisone (Sigma-Aldrich), $0.013 \mathrm{ng} / \mathrm{mL}$ triiodothyronine (SigmaAldrich), and $10 \mathrm{mM}$ nicotinamide (Sigma-Aldrich). The medium was replaced three times per week. Cells were maintained at $37^{\circ} \mathrm{C}$ under a $5 \% \mathrm{CO}_{2}$ atmosphere.

2.10. Western Blotting. Cells were washed with ice-cold PBS and lysed with RIPA buffer $(150 \mathrm{mM} \mathrm{NaCl}, 50 \mathrm{mM}$ Tris$\mathrm{HCl} \mathrm{pH} 7.4,1 \%$ Triton X-100, 0.1\% SDS, $25 \mathrm{mM} \mathrm{NaF}$, and $5 \mathrm{mM}$ EDTA with protease and phosphatase inhibitors; Pierce). Cell lysates were centrifuged at $450 \mathrm{~g}$ for $30 \mathrm{~min}$ at $4^{\circ} \mathrm{C}$ and supernatants were retrieved. Protein concentrations were determined by the BCA assay (Pierce Biotechnology). Proteins were separated on SDS-polyacrylamide gel and transferred electrophoretically to polyvinylidene difluoride (PVDF) membranes (Bio-Rad Laboratories) for immunoblotting. All primary antibodies were used at a dilution of 1:500. Immunoblotted bands were detected by enhanced chemiluminescence (ECL) with West Femto chemiluminescent substrate (Thermo Scientific) using the ChemiDoc MP Imaging System (Bio-Rad Laboratories). Image analysis was performed using Image Lab 5.2 software (Bio-Rad Laboratories).

2.11. Flow Cytometry Measurement of Autolysosome Formation. Cells transfected with the Premo ${ }^{\mathrm{TM}}$ Autophagy Tandem Sensor RFP-GFP-LC3B Kit were detached from the cell culture plate using TrypLE ${ }^{\mathrm{TM}}$ Express (Gibco), washed with ice-cold PBS, and stained with LIVE/DEAD ${ }^{\mathrm{TM}}$ Fixable Aqua Dead Cell Stain Kit (Invitrogen) for $30 \mathrm{~min}$ on ice following the manufacturer's recommendation. Cells were then washed once with PBS and resuspended in FACS buffer (PBS with $2 \% \mathrm{FBS}$ and $0.1 \% \mathrm{NaN}_{3}$ ). An Attune ${ }^{\mathrm{TM}} \mathrm{NxT}$ Flow Cytometer (Invitrogen) was used to perform flow cytometry. Data were analyzed using FlowJo software (BD Biosciences). The appropriate forward/side scatter profile was used to selectively include singlet cells in the analysis. Nonviable cells were excluded from the analysis by gating on cells unstained with LIVE/DEAD ${ }^{\text {TM }}$ Fixable Aqua Dead Cell Stain. Only the cells expressing the RFP-GFP-LC3B tandem construct (high RFP signal) were included in the analysis.

2.12. Immunostaining. In poly-D-lysine-coated 4-well chamber slides (Corning), 50000 cells per chamber were cultured for $48 \mathrm{~h}$, treated, fixed with $4 \%$ paraformaldehyde solution for $15 \mathrm{~min}$ at $37^{\circ} \mathrm{C}$, permeabilized with $0.2 \%$ Triton $\mathrm{X}-100$ in PBS for $10 \mathrm{~min}$, and blocked with $3 \%$ normal goat serum in PBS for $30 \mathrm{~min}$. Cells were then incubated overnight at $4^{\circ} \mathrm{C}$ with primary antibodies for LAMP1, LC3B, STX17, or TOMM20 (all 1:200) or 3 hours at room temperature for ZO-1 (1:100) immunostaining. After washing, cells were incubated with secondary antibodies and Hoechst 33342 $(1 \mu \mathrm{g} / \mathrm{mL})$ for $1 \mathrm{~h}$. Immunostainings were observed using a ZEISS LSM 700 confocal microscope (LAMP1, LC3B, STX17, and TOMM20) or a ZEISS Axio Observer (ZO-1).

2.13. Colocalization Analysis. Manders' colocalization coefficient analysis was performed using JACoP (Just Another Colocalization Plugin) in ImageJ software (NIH Image).

2.14. Statistical Analysis. Statistical analyses were performed using GraphPad Prism 8.0 software (GraphPad Software). One-way analysis of variance (ANOVA) with Sidak's multiple comparison test was performed after confirming the normality of residuals by the D'Agostino-Pearson test. Data are presented as mean \pm standard deviation (SD). A $p$ value lower than 0.05 was considered statistically significant. All comparisons made and corresponding $p$ values are presented in supplementary materials (Table S2).

\section{Results}

3.1. Sodium Iodate Caused a Concentration-Dependent Cytotoxicity on hTERT RPE-1 Cells. The human telomerase 
reverse transcriptase subunit-immortalized RPE cell line hTERT RPE- 1 has previously been used to study the impact of oxidative stress on RPE cell energy metabolism [30], as well as the cytoprotective role of autophagy in the RPE [31]. Oxidative damage to hTERT RPE-1 cells was induced using $\mathrm{NaIO}_{3}$ as oxidant. After treatment with $\mathrm{NaIO}_{3}$ for $2 \mathrm{~h}$, the cells exhibited a concentration-dependent increase in ROS accumulation (Figure 1(a)). The colorimetric reaction of CCK-8, a tetrazolium salt used to measure cellular dehydrogenase activities as an indicator of viability [32], revealed a concentration-dependent decrease in cellular viability after $24 \mathrm{~h}$ (Figure 1(b)). An increase in the percentage of cells positive for active caspase 3/7 was suggestive of apoptosis and preceded cell death (Figure 1(c)).

3.2. MPE-001 Protected hTERT RPE-1 Cells against $\mathrm{NaIO}_{3^{-}}$ Induced Cytotoxicity. The protective effect of the CD36 ligand MPE-001 on RPE cell oxidative damage was ascertained by a pretreatment with the azapeptide for $2 \mathrm{~h}$, followed by exposure to $\mathrm{NaIO}_{3}$. Treatment with MPE-001 alone did not impact the colorimetric reaction of CCK-8. MPE-001 improved the viability of hTERT RPE-1 cells exposed to 6.25 and $12.5 \mathrm{mM} \mathrm{NaIO}_{3}$ (Figure 2(a)). The $12.5 \mathrm{mM}$ concentration of $\mathrm{NaIO}_{3}$, against which MPE-001 had the most significant effect, was employed in subsequent experiments. MPE-001 caused $\sim 0.57$-fold and 0.73-fold decreases of $\mathrm{NaIO}_{3}$-induced ROS formation (Figure 2(b)) and apoptotic cell death (Figures 2(c) and 2(d)), respectively. The mitochondrial membrane potential is known to be disrupted during early apoptosis [33] and was assessed to confirm the effect of MPE-001 on apoptosis. Monomeric JC-1 dye has a cationic nature and green fluorescence. Upon accumulation in healthy mitochondria, JC-1 forms aggregates which emit a red fluorescence. Treatment with the oxidative phosphorylation uncoupler FCCP as positive control for $4 \mathrm{~h}$ caused a decrease in JC-1 red aggregates in all cells, indicative of mitochondrial depolarization. A visible loss of red fluorescence in apoptotic cells was detected after exposure to $\mathrm{NaIO}_{3}$ for $4 \mathrm{~h}$. In contrast, red fluorescence persisted and barely any apoptosis was observed in cells pretreated with MPE-001 prior to exposure to the oxidant (Figures 2(e) and 2(f) and Figure S1).

The CD36 dependence of the protective effect of MPE001 was verified using RNA interference. After $72 \mathrm{~h}$, knockdown with siRNA decreased CD36 mRNA expression by $88 \%$ (Figure 2(g)) and the cytoprotective effect of MPE-001 was completely abolished (Figure $2(\mathrm{~h})$ ).

3.3. MPE-001 Protected Differentiated ARPE-19 Cells against $\mathrm{NaIO}_{3}$-Induced Cytotoxicity. The human cell line ARPE-19 was used to validate the effect of MPE-001 on a differentiated and polarized RPE cell culture model. Culture of confluent ARPE-19 cells in low-serum medium has been shown to recapitulate some characteristics of native RPE cells, including the cobblestone morphology and expression of RPEspecific genes. Moreover, a polarized ARPE-19 cell monolayer can be promoted using the Transwell culture system [29]. Differentiated ARPE-19 cells grown on a Transwell insert displayed lateral distribution of zonal occludens-1 (ZO-1) suggestive of tight junction formation (Figure S2A)

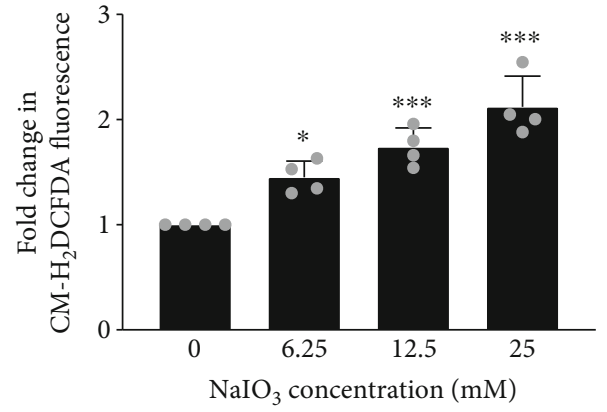

(a)

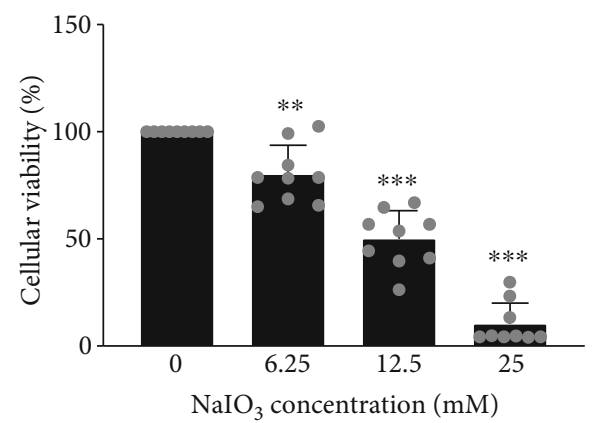

(b)

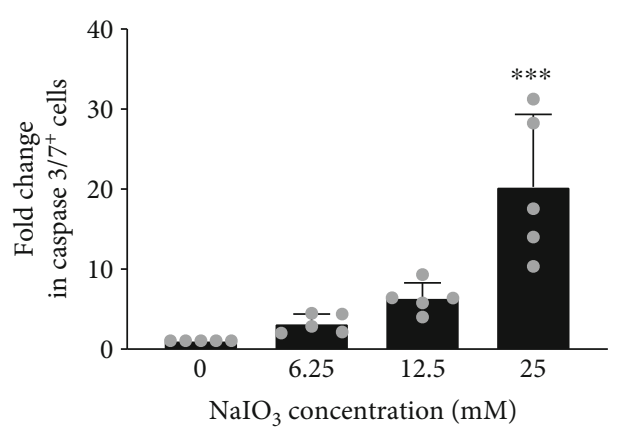

(c)

FIGURE 1: $\mathrm{NaIO}_{3}$ induced concentration-dependent ROS formation and death of hTERT RPE-1 cells. hTERT RPE- 1 cells were treated with increasing concentrations of $\mathrm{NaIO}_{3}$. (a) Cellular ROS after $2 \mathrm{~h}$ relative to vehicle-treated cells $(n=4)$. (b) Cellular viability assessed by CCK- 8 after $24 \mathrm{~h}(n=9)$. (c) Proportion of cells positive for active caspase $3 / 7$ after $4 \mathrm{~h}$ presented as fold change relative to vehicle-treated cells $(n=5)$. Mean $\pm \mathrm{SD},{ }^{*} p<0.05,{ }^{* *} p<$ 0.01 , and ${ }^{* * *} p<0.001$ vs vehicle.

and a similar loss of cellular viability to hTERT RPE-1 cells when exposed to $12.5 \mathrm{mM} \mathrm{NaIO}_{3}$ for $24 \mathrm{~h}$ (Figure S2B). The lower compartment of the Transwell culture system was pretreated with MPE-001, because CD36 expression has been mainly observed at the basal surface of rat RPE cells [34]. MPE-001 protected differentiated ARPE-19 cells from $\mathrm{NaIO}_{3}$ and improved cellular viability from $62 \%$ to $93 \%$ (Figure S2B).

3.4. The Protective Effect of MPE-001 Was Not Mediated by a Transcriptional Upregulation of Antioxidant Enzyme Expression. The importance of antioxidant enzyme expression on the antioxidant mechanism of MPE-001 action was ascertained using qRT-PCR. Neither $\mathrm{NaIO}_{3}$ nor MPE001 

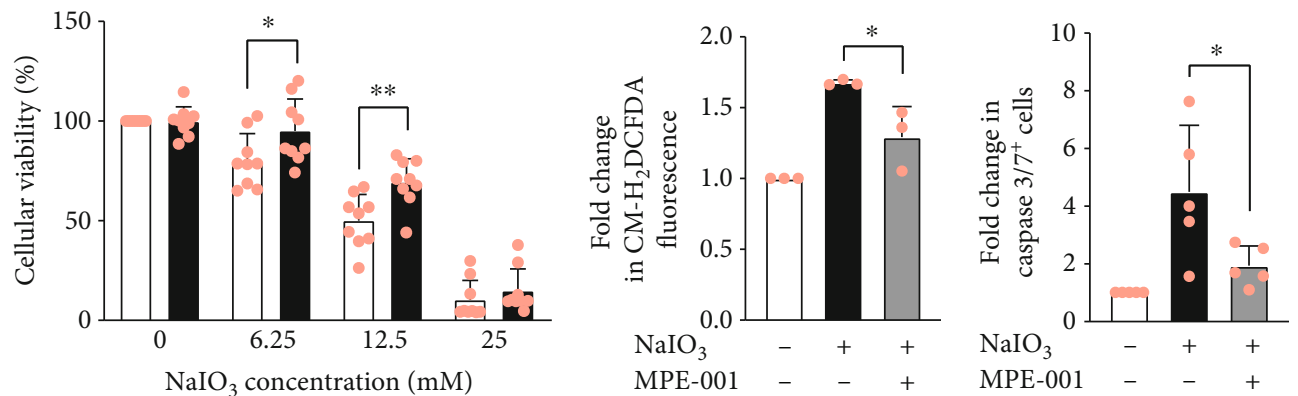

$\square$ Vehicle
MPE-001

(a)

(b)

(c)
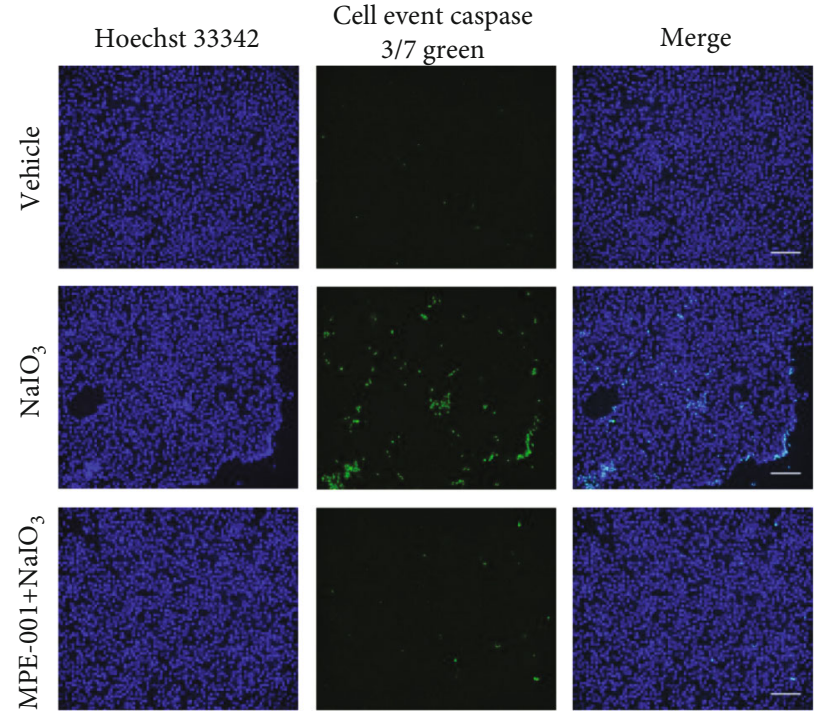

(d)

\section{Vehicle}

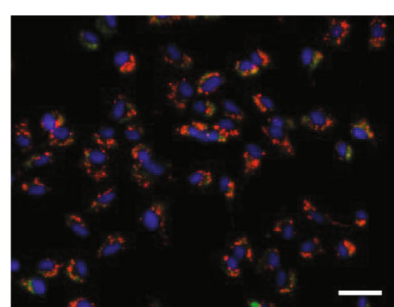

$\mathrm{NaIO} 3$

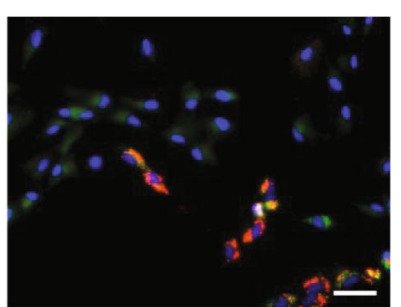

MPE- $001+\mathrm{NaIO}_{3}$

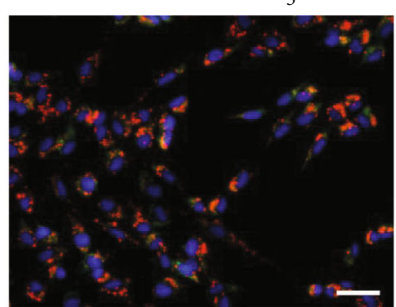

(e)

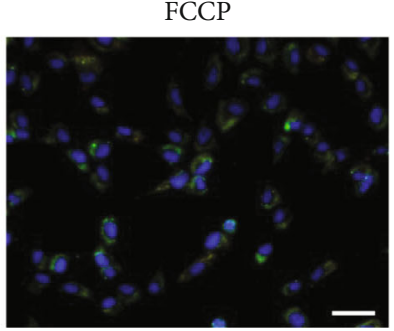

Figure 2: Continued. 


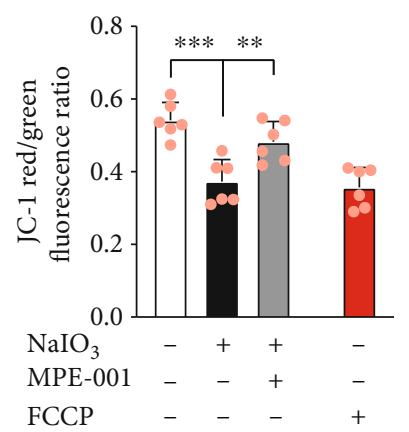

(f)

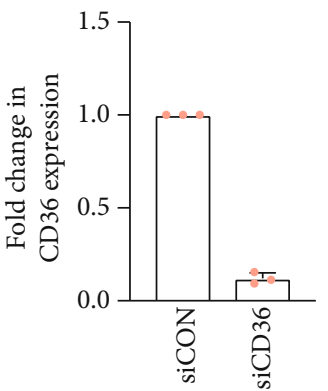

(g)

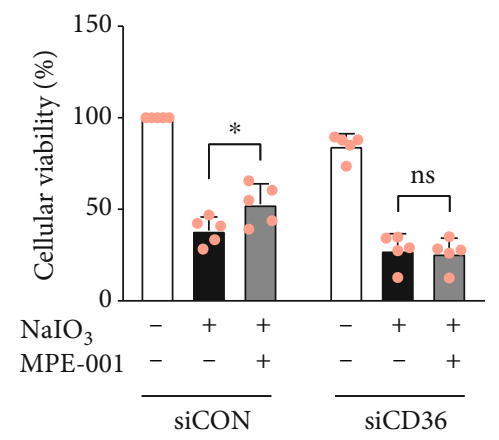

(h)

FIGURE 2: MPE-001 prevented $\mathrm{NaIO}_{3}$-induced ROS formation and apoptosis of hTERT RPE-1 cells. hTERT RPE- 1 cells were pretreated with $1 \mu \mathrm{M}$ MPE-001 for $2 \mathrm{~h}$ and then exposed to $\mathrm{NaIO}_{3}$. (a) Cellular viability assessed by CCK- 8 after $24 \mathrm{~h}$ of $\mathrm{NaIO}_{3}$ treatment $(n=9)$. (b) Cellular ROS after $2 \mathrm{~h}$ of $12.5 \mathrm{mM} \mathrm{NaIO}_{3}$ treatment presented as fold change relative to vehicle-treated cells $(n=3)$. (c) Proportion of cells positive for active caspase $3 / 7$ after $4 \mathrm{~h}$ of $12.5 \mathrm{mM} \mathrm{NaIO}_{3}$ treatment presented as fold change relative to vehicle-treated cells $(n=5)$. (d) Representative images of CellEvent Caspase-3/7 Green and Hoechst 33342 staining (scale bar $=200 \mu \mathrm{m}$ ). (e) Representative images of JC-1 staining (JC-1 aggregate: red; JC-1 monomer: green; Hoechst 33342: blue; scale bar $=50 \mu \mathrm{m}$ ). (f) Red to green fluorescence ratio of JC-1 after $4 \mathrm{~h}$ of $12.5 \mathrm{mM} \mathrm{NaIO}_{3}$ treatment. $5 \mu \mathrm{M}$ FCCP was used as control $(n=6)$. (g) CD36 mRNA expression $(n=3)$ and $(\mathrm{h})$ cellular viability assessed by CCK- 8 after $24 \mathrm{~h}$ of $12.5 \mathrm{mM} \mathrm{NaIO}$ treatment $(n=5)$ of cells incubated for $72 \mathrm{~h}$ with either $20 \mathrm{nM}$ control siRNA (siCON) or CD36 siRNA (siCD36). Mean $\pm \mathrm{SD}$, ns: nonsignificant, ${ }^{*} p<0.05,{ }^{* *} p<0.01$, and ${ }^{* * *} p<0.001$.

exhibited effect on the expression of CAT, GPX1, SOD1, SOD2, PRDX3, PRDX5, and TXN2 (Figure 3(a)). Treatment with $\mathrm{NaIO}_{3}$ for $4 \mathrm{~h}$ increased expression of genes targeted by the transcription factor NRF2 (or NFE2L2): HMOX1, NQO1, GCLM, PRDX1, and TXNRD1 (Figure 3(a)). Moreover, exposure to $\mathrm{NaIO}_{3}$ increased expression of NFE2L2 (Figure 3(b)), which is a master regulator of the antioxidant system [35]. On the contrary, MPE-001 had no effect on the expression of NFE2L2, NFE2L2-targeted genes, nor HMOX1 protein at different timepoints after $\mathrm{NaIO}_{3}$ treatment (Figures 3(a)-3(c)). The protective effect of MPE-001 was therefore not likely mediated by a transcriptional upregulation of antioxidant enzymes in the RPE cell model.

3.5. MPE-001 Improved Autophagic Flux in $\mathrm{NaIO}_{3}$-Treated hTERT RPE-1 Cells and Its Cytoprotective Effect Was Autophagy Dependent. The relevance of autophagy in the antioxidative and cytoprotective mechanisms of MPE-001 action was next investigated because of the absence of an effect on antioxidant gene expression. Microtubuleassociated protein light chain 3B (LC3B) is a structural protein of autophagosomal membranes. During autophagy, the cytosolic form of LC3B (LC3-I) undergoes lipidation to form LC3-II which is integrated into growing phagophores [36]. Consequently, the LC3-II/I ratio is a discerning indicator of autophagy [37]. Treatment with $\mathrm{NaIO}_{3}$ for $4 \mathrm{~h}$ induced a statistically significant LC3-II/I ratio increase compared to vehicle-treated cells, which was not observed when all cells were concomitantly treated with the inhibitor of lysosomal acidification bafilomycin A1 (Figure 4(a)). The latter LC3II/I ratios were unchanged and increased by pretreatment with MPE-001 in the absence and presence, respectively, of bafilomycin A1. Relative to vehicle controls, combined treatment with $\mathrm{NaIO}_{3}$ and MPE-001 induced 2.49- and 3.50-fold increases in the LC3-II/I ratio in the absence and presence, respectively, of bafilomycin A1 (Figure 4(a)), suggestive of increased autophagosome biogenesis and degradation. Similar trends were observed in the LC3-II/GAPDH ratio (Figure 4(a)). These data indicate an inefficient clearing of autophagosomes in $\mathrm{NaIO}_{3}$-treated cells, which is improved by MPE001 pretreatment. MPE-001 treatment alone did not alter the LC3-II/I ratio (Figure 4(a)).

The effects of MPE-001 on autophagic flux were further validated using hTERT RPE-1 cells expressing an RFPGFP-LC3B tandem construct. To monitor autolysosome formation, the quenching of acid-labile green fluorescent protein (GFP) signal relative to the acid-stable red fluorescent protein (RFP) signal was measured by flow cytometry. Cells with high autophagic flux (high RFP/GFP ratio) were defined using cells starved for 1 hour in Hank's balanced salt solution (HBSS) (Figure 4(b)). Treatment with $\mathrm{NaIO}_{3}$ or bafilomycin A1 for 4 hours reduced autophagic flux, decreasing the percentage of cells with high autophagic flux from $18 \%$ to $13 \%$ or $10 \%$, respectively. Treatment with MPE-001 alone did not induce autolysosome formation but increased the percentage of cells with high autophagic flux in $\mathrm{NaIO}_{3}$ exposed cells to $21 \%$ (Figure 4(c)).

Autophagy inhibitors wortmannin and bafilomycin A1 both abolished the cytoprotective effect of MPE-001 on hTERT RPE-1 cells (Figure 4(d)). In contrast, bafilomycin A1 had no effect on the improvement of the viability of $\mathrm{NaIO}_{3}$-treated cells that was produced by the antioxidant NAC, which is a synthetic precursor of L-cysteine and an elevator of glutathione biosynthesis (Figure S3A, B). The cytoprotective effect of MPE-001 is thus mediated by autophagy and not through the direct scavenging of $\mathrm{NaIO}_{3}$-generated ROS. Similarly, the cytoprotective effect of MPE-001 against $\mathrm{NaIO}_{3}$ on differentiated ARPE-19 cells was abolished by bafilomycin A1, suggesting an autophagy-dependent protective mechanism in the cultured system (Figure S4). 

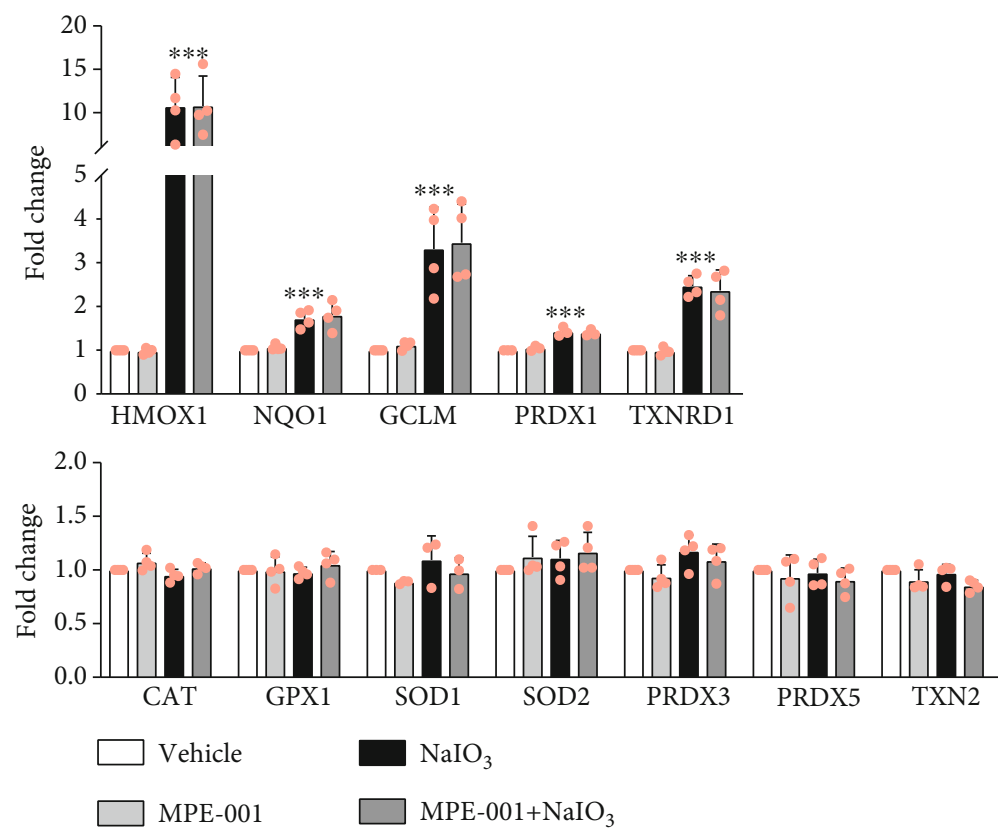

(a)

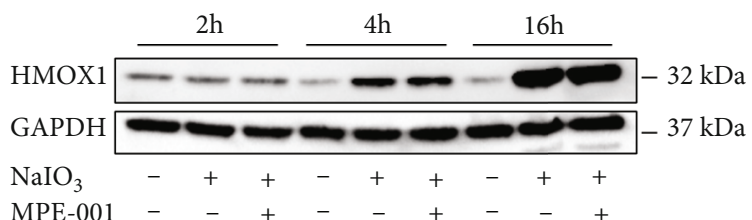

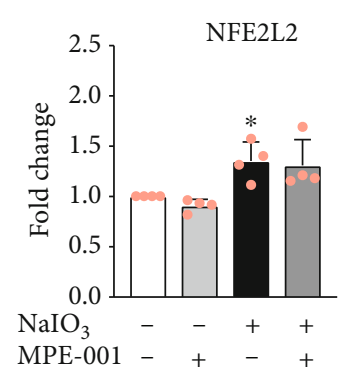

(b)

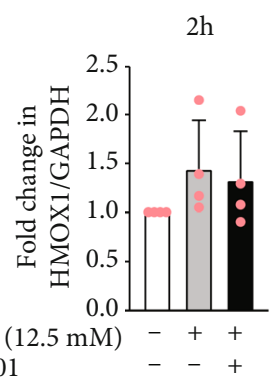

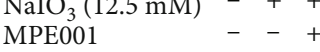

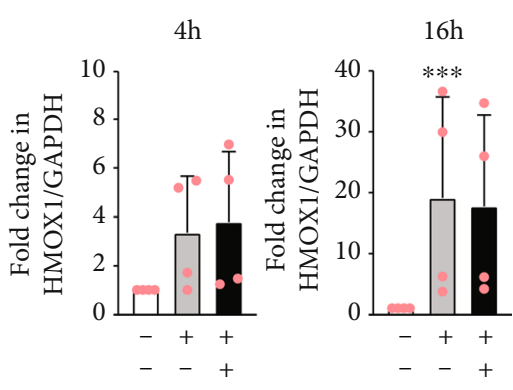

(c)

FIgURE 3: The effect of $\mathrm{NaIO}_{3}$ and/or MPE-001 treatment on antioxidant enzyme expression. hTERT RPE-1 cells were pretreated with $1 \mu \mathrm{M}$ MPE-001 for $2 \mathrm{~h}$ and then exposed to $12.5 \mathrm{mM} \mathrm{NaIO}_{3}$. (a) mRNA expression of antioxidant enzymes and (b) NFE2L2 expression after $4 \mathrm{~h}$ of $\mathrm{NaIO}_{3}$ treatment. Data are presented as fold change relative to vehicle controls. $n=3-4$, mean $\pm \mathrm{SD},{ }^{*} p<0.05$ and ${ }^{* * *} p<0.001$ vs vehicle. (c) Immunoblot of HMOX1 and GAPDH (upper) and relative quantification of HMOX1/GAPDH (lower) at different time points following $\mathrm{NaIO}_{3}$ treatment. $n=4$, mean $\pm \mathrm{SD}$, and ${ }^{* * *} p<0.001$ vs vehicle.

3.6. MPE-001 Improved Autophagosome Maturation in $\mathrm{NaIO}_{3}$-Treated hTERT RPE-1 Cells. Syntaxin 17 (STX17) is a soluble $N$-ethylmaleimide-sensitive factor attachment protein receptor (SNARE) required for autophagosomelysosome fusion. Mature autophagosomes that are primed to fuse with lysosomes are LC3B and syntaxin 17 (STX17) positive [38]. The restorative effect of MPE-001 on $\mathrm{NaIO}_{3}$ induced inhibition of autophagic flux was examined in transfected cells expressing LC3B-GFP by immunostaining for STX17. Colocalization of LC3B-GFP puncta and STX17 immunostaining assessed in the presence of bafilomycin A1 was scarce in $\mathrm{NaIO}_{3}$-treated cells (Figure 5(a)), indicating a defect in autophagosomal maturation. The proportion of LC3B puncta that colocalize with STX-17 immunostaining was significantly increased in MPE-001-pretreated cells (Figures 5(a) and 5(b)). The $\mathrm{NaIO}_{3}$ treatment resulted in STX17 accumulation outside of autophagosomes (Figure 5(a)). Western blotting indicated that the increase in STX17 expression was only statistically significant in the MPE-001-pretreated cells exposed to $\mathrm{NaIO}_{3}$ (Figure 5(c)).

3.7. MPE-001 Prevented $\mathrm{NaIO}_{3}$-Induced Mitochondrial Superoxide Production through Autophagy Enhancement. Mitochondria are both a target and a major source of ROS. 


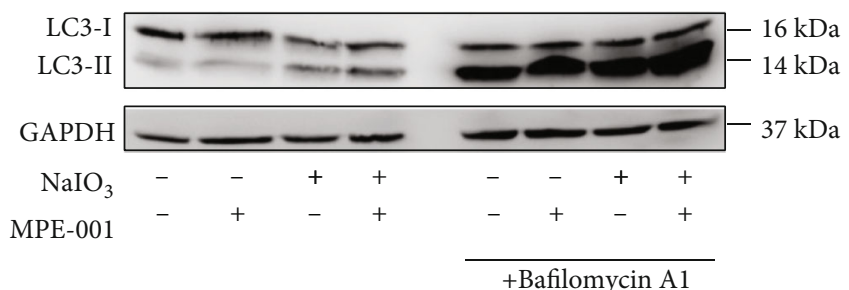

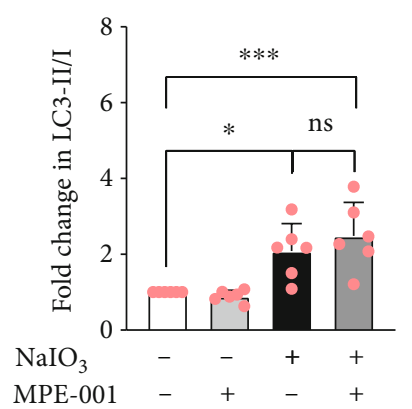

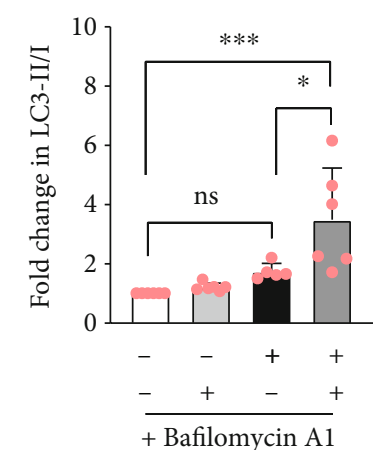

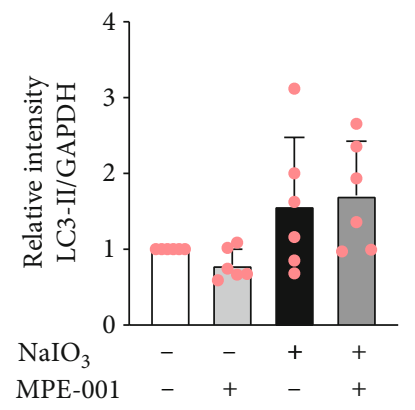

MPE-00

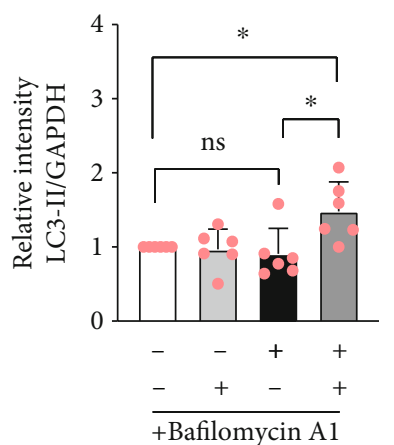

(a)
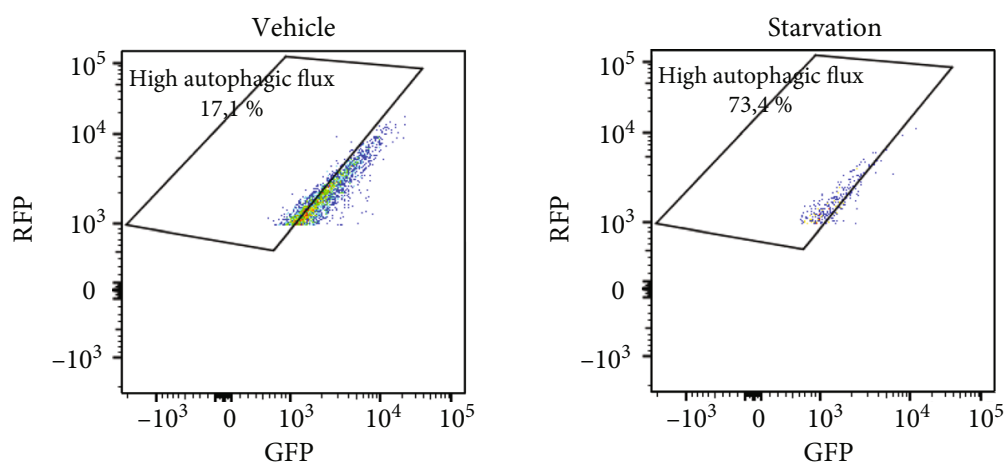

(b)

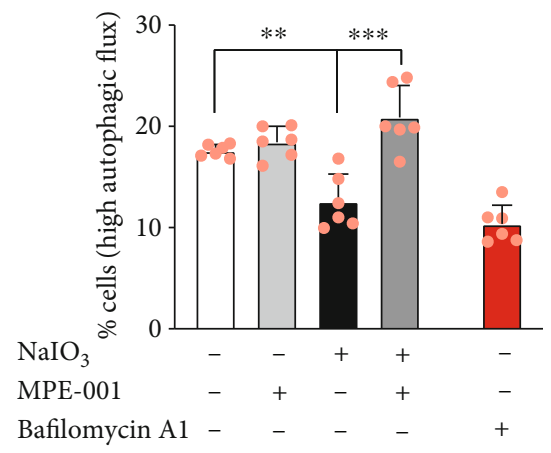

(c)
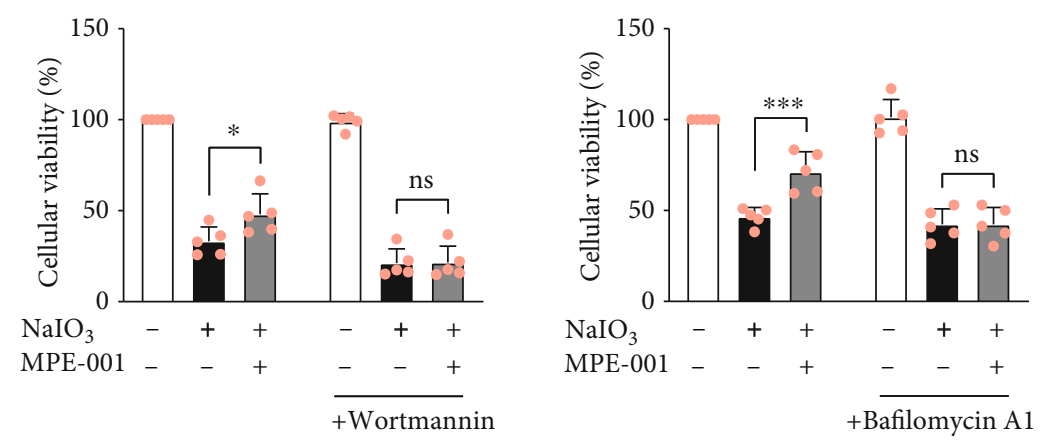

(d)

FIgURE 4: MPE-001 enhanced autophagy in $\mathrm{NaIO}_{3}$-treated cells. hTERT RPE-1 cells were pretreated with $1 \mu \mathrm{M}$ MPE-001 for $2 \mathrm{~h}$ and then exposed to $12.5 \mathrm{mM} \mathrm{NaIO}_{3}$ for $4 \mathrm{~h} \mathrm{(a,} \mathrm{c,} \mathrm{and} \mathrm{d)} \mathrm{or} \mathrm{treated} \mathrm{with} 10 \mathrm{nM}$ bafilomycin for 6 hours (c) or starved in HBSS for 1 hour (b). (a) Immunoblot of LC3B and GAPDH (upper) and quantification of LC3-II/LC3-I and LC3-II/GAPDH ratio (lower) relative to respective vehicle controls in the absence and presence of $10 \mathrm{nM}$ bafilomycin A1 $(n=6)$. (b, c) Flow cytometry analysis of RFP and GFP signals of cells transfected with an RFP-GFP-LC3B tandem construct. Example of gating used to identify the percentage of cells with high

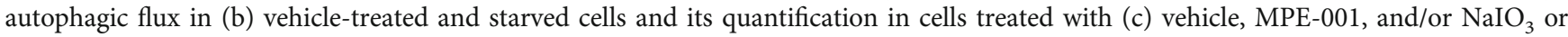
with bafilomycin A1 $(n=6)$. (d) Cellular viability assessed by CCK- 8 after treatment with $\mathrm{NaIO}_{3}$ for $24 \mathrm{~h}$ in the absence and presence of $100 \mathrm{nM}$ wortmannin (left, $n=5$ ) or $10 \mathrm{nM}$ bafilomycin A1 (right, $n=5$ ). Mean $\pm \mathrm{SD}$, ns: nonsignificant, ${ }^{*} p<0.05$, ${ }^{* *} p<0.01$, and ${ }^{* * *} p<0.001$. 


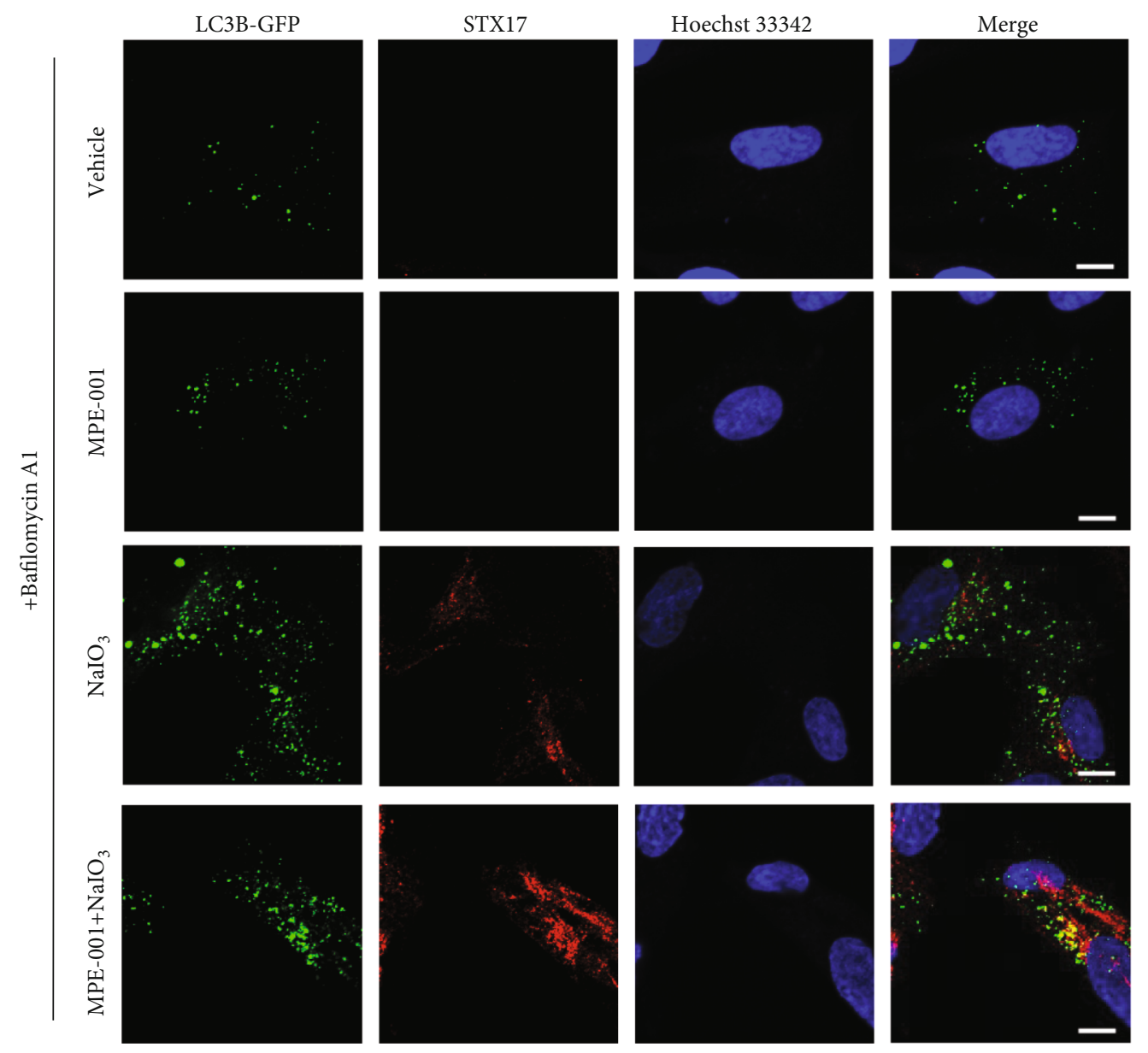

(a)

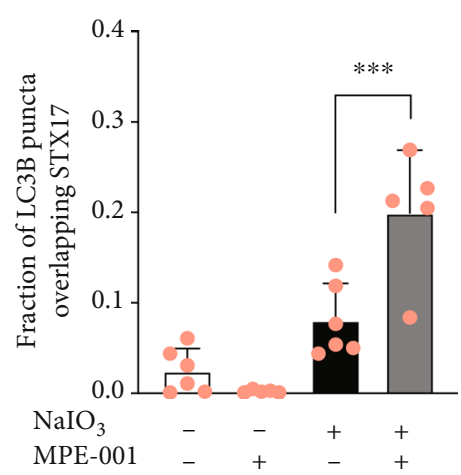

(b)
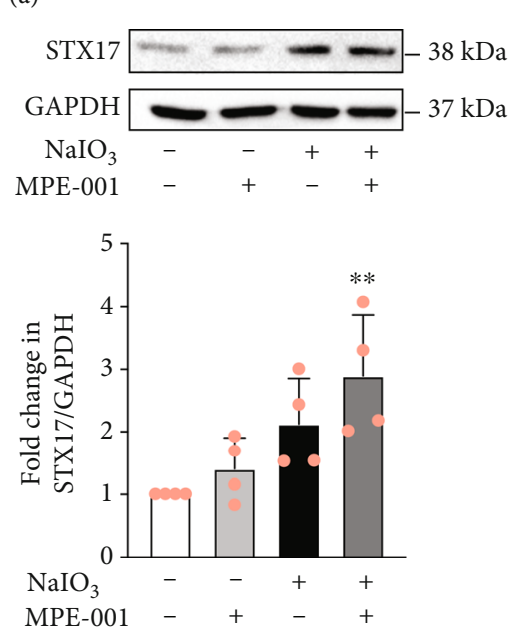

(c)

FIGURE 5: MPE-001 increased the recruitment of STX17 to autophagosomes in $\mathrm{NaIO}_{3}$-treated cells. hTERT RPE-1 cells were pretreated with $1 \mu \mathrm{M}$ MPE-001 for $2 \mathrm{~h}$ and then exposed to $12.5 \mathrm{mM} \mathrm{NaIO}_{3}$ for $4 \mathrm{~h}$. (a) Representative images of LC3B-GFP-transfected cells immunostained for STX17 following treatments in the presence of $10 \mathrm{nM}$ bafilomycin A1 (scale bar $=10 \mu \mathrm{m}$ ). The same gamma correction was applied to all images. (b) Fraction of LC3B puncta that overlap STX17 staining. Manders' colocalization coefficient analysis was carried out across a series of 5 to 6 images of 6 to 18 cells each. Mean \pm SD, ${ }^{* * *} p<0.001$. (c) Immunoblot of STX17 and GAPDH (upper) and relative quantification of STX17/GAPDH (lower). $n=4$, mean \pm SD, ns: nonsignificant, and ${ }^{* *} p<0.01$ vs vehicle. 


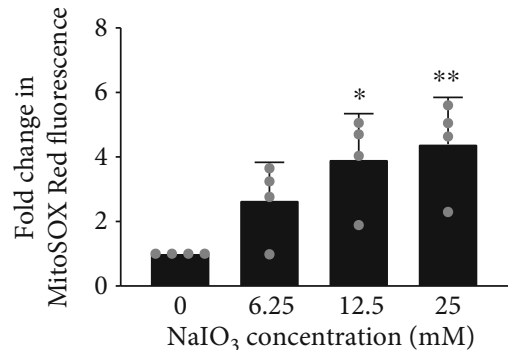

(a)
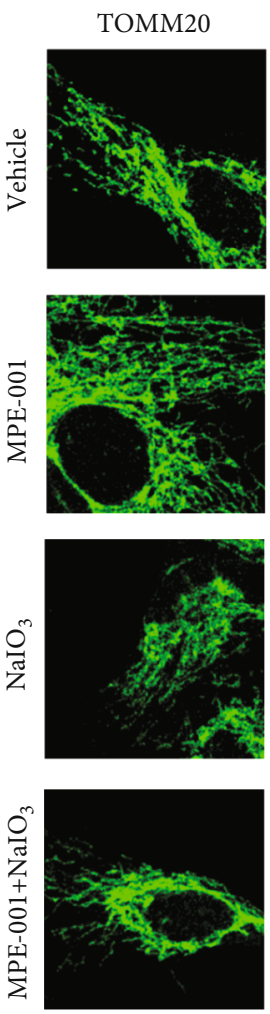
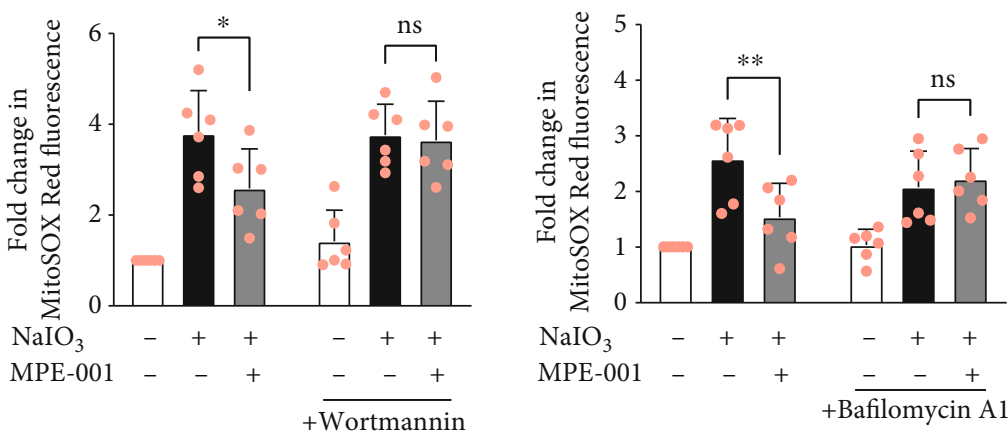

(b)
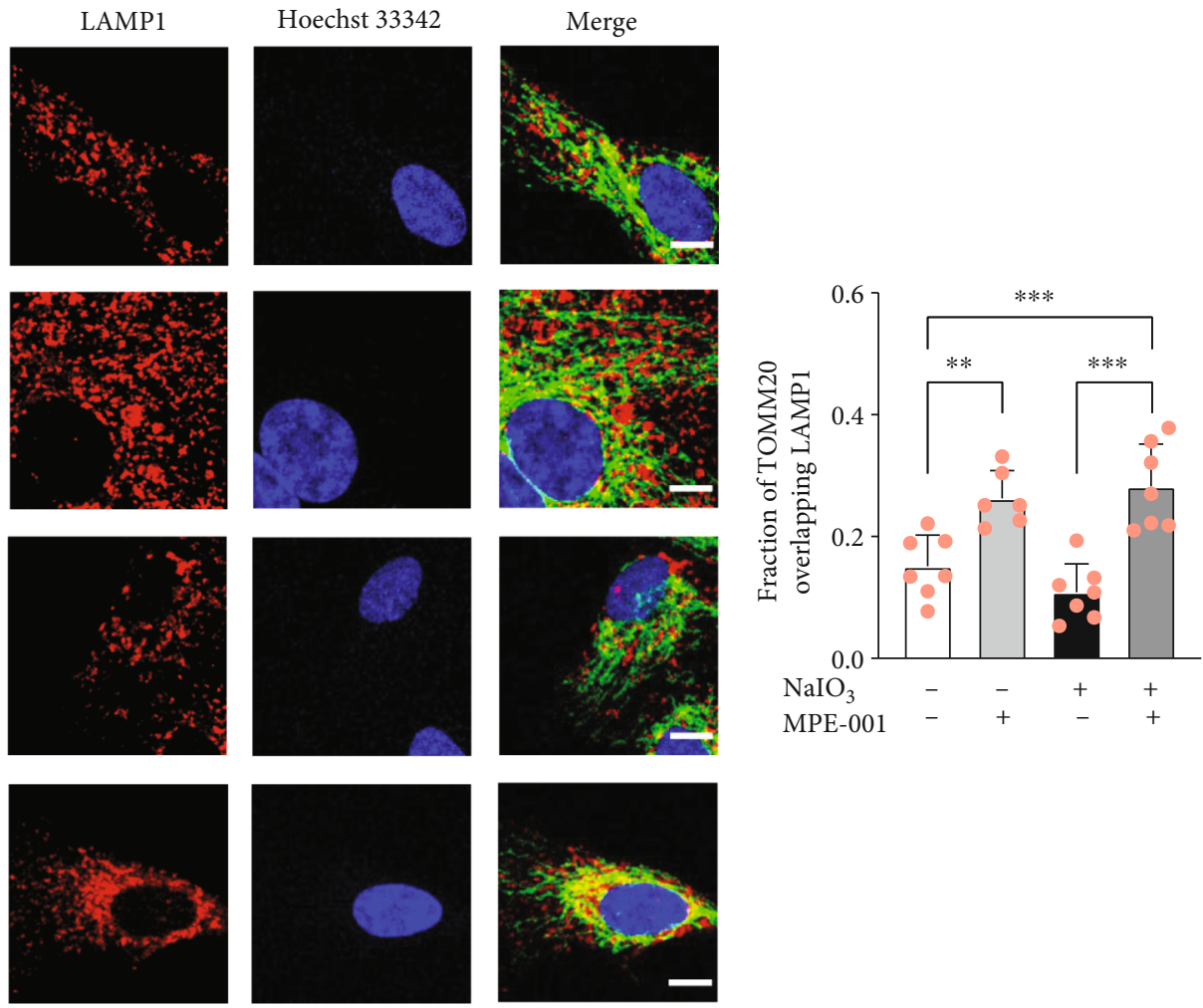

(c)

FIGURE 6: MPE-001 prevented $\mathrm{NaIO}_{3}$-induced mitochondrial superoxide formation in an autophagy-dependent manner. (a) Mitochondrial superoxide formation in hTERT RPE- 1 cells treated with increasing concentrations of $\mathrm{NaIO}_{3}$ for $2 \mathrm{~h}$ as fold change relative to vehicle-treated cells $(n=4) .{ }^{*} p<0.05$ and ${ }^{* *} p<0.01$ vs vehicle. $(\mathrm{b}, \mathrm{c})$ Cells were pretreated with $1 \mu \mathrm{M} \mathrm{MPE}-001$ for $2 \mathrm{~h}$ and then exposed to $12.5 \mathrm{mM}$ NaIO ${ }_{3}$. (b) Mitochondrial superoxide formation in the absence and presence of $100 \mathrm{nM}$ wortmannin (left, $n=6$ ) or $10 \mathrm{nM}$ bafilomycin A1 (right, $n=6$ ) after $2 \mathrm{~h}$ of $\mathrm{NaIO}_{3}$ treatment. Mean $\pm \mathrm{SD}$, ns: nonsignificant, ${ }^{*} p<0.05$, and ${ }^{* *} p<0.01$. (c) Representative images of TOMM20 and LAMP1 immunostaining after $4 \mathrm{~h}$ of $\mathrm{NaIO}_{3}$ treatment (scale bar $=10 \mu \mathrm{m}$ ). (d) Fraction of TOMM20 staining that overlaps with LAMP1 staining. Manders' colocalization coefficient analysis was carried out across a series of 6 to 7 images of 1 to 13 cells each. Mean \pm SD, ${ }^{* *} p<0.01$, and ${ }^{* * *} p<0.001$.

Improper removal of damaged mitochondria by autophagy results in excessive generation of ROS [39], which may be the causative agent in $\mathrm{NaIO}_{3}$-induced cell death. The effects of $\mathrm{NaIO}_{3}$ and MPE-001 on mitochondrial ROS formation were examined by measuring superoxide formation. After treatment with $\mathrm{NaIO}_{3}$ for $2 \mathrm{~h}$, mitochondrial superoxide increased in a concentration-dependent manner (Figure 6(a)). Pretreatment with MPE-001 decreased the formation of mitochondrial superoxide induced by $12.5 \mathrm{mM}$ $\mathrm{NaIO}_{3}$ (Figure 6(b)). Wortmannin and bafilomycin A1 both abolished the effect of MPE-001 on mitochondrial superoxide formation induced by $\mathrm{NaIO}_{3}$ (Figure 6(b)), indicating that the activity on ROS was secondary to the influence on autophagy. Immunostaining of the lysosomal-associated membrane protein 1 (LAMP1) and the translocase of outer mitochondrial membrane 20 (TOMM20) showed that MPE-001 treatment increased the colocalization of TOMM20-positive structures with LAMP1-positive structures, regardless of cell exposure to $\mathrm{NaIO}_{3}$ (Figures 6(c) and 6(d)). Immunostaining of LC3B and TOMM20 in the presence of bafilomycin A1 showed in 


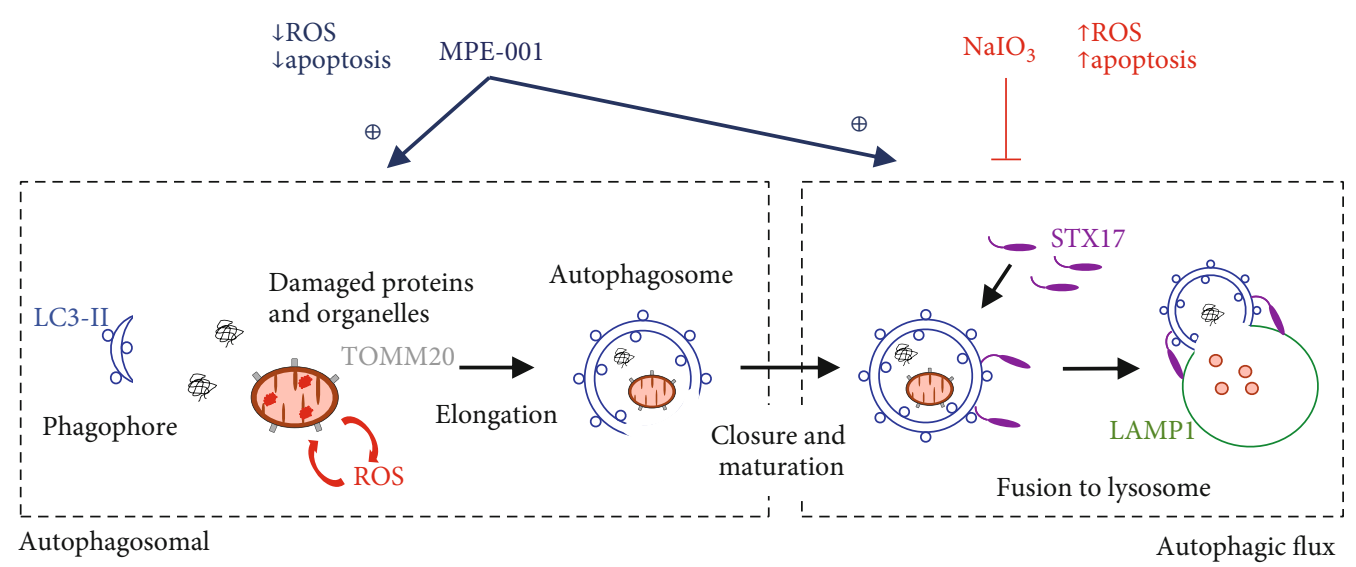

FIGURE 7: Underlying mechanism of the antioxidative and antiapoptotic effects of CD36 activation by MPE-001. Treatment of RPE cells with $\mathrm{NaIO}_{3}$ disrupts later steps of autophagic processes, leading to cell death. MPE-001 decreases ROS formation and apoptosis by promoting the closure and maturation of autophagosomes, which will recruit STX17 and fuse with lysosomes for the degradation of damaged proteins and organelles.

contrast that $\mathrm{NaIO}_{3}$ treatment, combined or not with MPE001, increased the recruitment of autophagosomes to mitochondria (Figure S5A, B), indicating that the oxidant inhibits autophagy after earlier steps of phagophore formation, elongation, and cargo engulfment.

The role of decreased mitochondrial ROS for the enhancement of survival of $\mathrm{NaIO}_{3}$-treated cells was explored using two mitochondria-selective ROS scavengers: MitoQ and MitoTEMPO, which are thought to insert into the inner mitochondrial membrane [40] and to cross the membrane and accumulate in the mitochondrial matrix, respectively [41]. Pretreatment with MitoTEMPO for $1 \mathrm{~h}$ failed to decrease mitochondrial superoxide formation (Figure S6A) and to prevent cell loss (Figure S6B). Pretreatment with MitoQ for $1 \mathrm{~h}$ decreased $\mathrm{NaIO}_{3}$-induced mitochondrial superoxide formation by $\sim 0.32$-fold (Figure S6C) but failed to improve cellular viability (Figure S6D). These results suggest that scavenging of mitochondrial ROS is insufficient to rescue $\mathrm{RPE}$ cells from $\mathrm{NaIO}_{3}$-induced cytotoxicity.

\section{Discussion}

A regiment composed of antioxidant supplements (vitamins $\mathrm{C}$ and $\mathrm{E}$, lutein, and zeaxanthin), zinc, and copper is currently recommended to AMD patients in an attempt to limit oxidative damage to the RPE and preserve photoreceptor integrity [42]. Such a regiment has however limited effect, except in advanced stages $[43,44]$, and cannot prevent disease onset [45]. New approaches are urgently needed for preventing and halting the pathology of AMD ideally at the earlier stages. CD36 is a promising therapeutic target due in part to the involvement in sterile inflammation and lipid deposition observed in dry AMD [46]. Previously, GHRP-6 derivatives have demonstrated beneficial effects as CD36 ligands that preserve photoreceptors in mouse models of AMD $[18,20]$. The potential of such CD36 ligands to mitigate oxidative stress and preserve RPE integrity has now been demonstrated.
The cytoprotective effect of the GHRP-6 derivative CD36 ligand MPE-001 against $\mathrm{NaIO}_{3}$-induced oxidative damage was revealed in human RPE cells in culture. The oxidant was shown to disrupt autophagic flux by a mechanism that entailed autophagosome formation without lysosome fusion. In spite the activation of the antioxidant system, oxidative stress and cell death persisted likely due to the inability of RPE cells to clear damaged proteins and organelles. The $\mathrm{NaIO}_{3}$-induced defect in autophagic flux was remedied by pretreatment of cells with MPE-001. Improvement in autophagic processes by MPE-001 resulted in decreased mitochondrial ROS accumulation and diminished apoptosis of RPE cells. In unstressed cells, MPE-001 treatment had no effect on the antioxidant system nor on autophagy.

Apoptosis mediated by caspase $3 / 7 / 8$ activation $[23,27]$ and necroptosis $[22,27,28]$ both have been reported to account for RPE cell death in culture treated with $\mathrm{NaIO}_{3}$. In accordance with the earlier studies, $\mathrm{NaIO}_{3}$ induced caspase 3/7 activation in hTERT RPE-1 cells. Moreover, $\mathrm{NaIO}_{3}$-induced cell death was accompanied by a disruption of the mitochondrial membrane potential as shown previously using JC-1 ratio-metric analysis and imaging $[23,26]$. MPE-001 was found to decrease $\mathrm{NaIO}_{3}$-induced ROS formation and specifically block mitochondrial depolarization and caspase 3/7-mediated apoptosis.

Strategies to boost the antioxidant system in RPE cells have been proposed to treat AMD $[26,47,48]$. For example, activation of NRF2 has been shown to protect RPE cells from oxidative stress both in vitro [26] and in vivo [49]. In contrast, MPE-001 did not upregulate antioxidant enzyme expression at the studied time points. The effect of MPE001 on ROS formation was observed as early as $2 \mathrm{~h}$ after $\mathrm{NaIO}_{3}$ treatment, opposing a mechanism implicating de novo transcription of antioxidant enzymes.

Autophagy has been shown to mitigate the impact of oxidative stress in the RPE. After $\mathrm{NaIO}_{3}$ treatment, markers of autophagy increased in ARPE-19 cells [24-27] and enhanced autophagic flux protected RPE cells from oxidative stress induced by $\mathrm{NaIO}_{3}$ [26] and $\mathrm{H}_{2} \mathrm{O}_{2}$ [50]. Blockade of 
autophagy by 3-methyladenine or respective knockdowns of ATG7 and Beclin-1 all have been observed to exacerbate the oxidative and cytotoxic effects of $\mathrm{H}_{2} \mathrm{O}_{2}$ [50]. In sum, autophagy has been shown to play a crucial role in RPE cell defense against oxidative damage.

MPE-001 protected RPE cells against $\mathrm{NaIO}_{3}$-induced oxidative damage by improving autophagic processes. The effects of MPE-001 were completely abolished by wortmannin or bafilomycin A1. Only after complete autophagosome closure does the autophagosome outer membrane recruit STX17, which is not observed in intermediate unclosed states [51]. Moreover, depletion of STX17 caused an accumulation of autophagosomes without degradation [51]. In this study, few autophagosomes were STX17 positive in $\mathrm{NaIO}_{3}$-treated cells, indicating a defect in autophagosome maturation and fusion to lysosomes. Pretreatment with MPE-001 remedied the effect of $\mathrm{NaIO}_{3}$ on autophagosome maturation and increased the proportion of LC3B puncta that colocalize with STX17 immunostaining. A role of CD36 in autophagy promotion by secreted glycoprotein CD5 antigen-like protein (CD5L) has been reported in macrophages [52] and in a model of hepatic ischemic/reperfusion injury [53]. In the latter, CD5L was shown to have antiapoptotic and antioxidative effects in a CD36- and Atg7-dependent manner [53]. The CD36 ligand CD5L is expressed in RPE cells and a high amount of circulating CD5L has been observed in some AMD patients [54]. The roles of CD5L in normal RPE cell physiology and AMD pathology have yet to be elucidated.

Increased mitochondrial superoxide formation has previously been associated with the cytotoxic effect of $\mathrm{NaIO}_{3}$ [23] as observed in this study. MPE-001 decreased $\mathrm{NaIO}_{3}$-induced mitochondrial superoxide production in an autophagy-dependent manner. On the contrary, scavenging of mitochondrial ROS by MitoQ did not rescue hTERT RPE-1 cells. Restored autophagic flux with clearance of defective mitochondria and cytosolic damaged proteins may account for the preventive effect of MPE-001 on mitochondrial depolarization and apoptosis which was likely not attained by the ROS scavenger MitoQ. Autophagy inhibition by bafilomycin A1 in combination with tert-butyl hydroperoxide-induced mitochondrial oxidative insult, but not bafilomycin A1 alone, has previously been shown to induce mitochondrial depolarization and cell death [55]. Autophagic processes are therefore crucial in maintaining mitochondrial health in cells subjected to oxidative stress. Curiously, MPE-001 treatment alone increased the colocalization of TOMM20-positive mitochondria with LAMP1-positive lysosomes without increasing TOMM20 colocalization with LC3B. Treatment of the cells with MPE-001 might be accelerating mitochondria turnover in healthy cells in an autophagy-independent anner. Impaired lysosomal removal of damaged mitochondria has been theorized to contribute to cell death associated with aging [56]. Moreover, mitochondria in the RPE of AMD patients exhibit more pronounced age-associated alterations in morphology and activity $[57,58]$. The improved autophagic activity resulting from CD36 activation may have broad benefits in a wide range of age-related diseases including AMD.

\section{Conclusion}

In summary, the CD36 ligand MPE-001 had antioxidative and antiapoptotic effects on RPE cells by a mechanism featuring restoration of autophagic flux (Figure 7). Modulation of the RPE redox status by pharmacological targeting of CD36 was evidenced for the first time. Combined with their known anti-inflammatory effect, the ability of azapeptide CD36 ligands to modulate oxidative stress offers potential for preserving RPE and photoreceptor integrity and a novel strategy for remedying the pathogenesis of dry AMD.

\section{Data Availability}

The data used to support the findings of this study are available upon request.

\section{Conflicts of Interest}

The authors declare that there is no conflict of interest regarding the publication of this paper.

\section{Acknowledgments}

This work was supported by the Canadian Institutes of Health Research (CIHR) (PPP-90157) and the Natural Sciences and Engineering Research Council (NSERC)-CIHR Collaborative Health Research Project "Azacyclopeptide Modulators of Immuno-metabolism to Treat Age-Related Macular Degeneration" (163973). M-FD received financial supports from CIHR, Fonds de recherche Santé du Québec (FRQS), the Japan Society for the Promotion of Science (JSPS), and Mitacs Globalink.

\section{Supplementary Materials}

contains Tables S1-S2 and Figures S1-S6. Table S1: primer sequences. Table S2: statistical comparisons and $p$ values. Figure S1: MPE-001 pretreatment prevents the $\mathrm{NaIO}_{3}$ induced loss of mitochondrial membrane potential. Figure S2: MPE-001 improved the viability of differentiated ARPE19 cells exposed to $\mathrm{NaIO}_{3}$. Figure S3: NAC protected hTERT RPE-1 cells against $\mathrm{NaIO}_{3}$-induced cytotoxicity in an autophagy-independent manner. Figure S4: bafilomycin A1 abolished the cytoprotective effect of MPE-001 on differentiated ARPE-19 cells. Figure S5: the effect of MPE-001 and/or $\mathrm{NaIO}_{3}$ treatments on LC3B and TOMM20 colocalization. Figure S6: the effect of mitochondria-selective antioxidant compounds on $\mathrm{NaIO}_{3}$-induced cytotoxicity. (Supplementary materials)

\section{References}

[1] S. Datta, M. Cano, K. Ebrahimi, L. Wang, and J. T. Handa, "The impact of oxidative stress and inflammation on RPE degeneration in non- neovascular AMD," Progress in Retinal and Eye Research, vol. 60, pp. 201-218, 2017.

[2] D. Pascolini and S. P. Mariotti, "Global estimates of visual impairment: 2010," The British Journal of Ophthalmology, vol. 96, no. 5, pp. 614-618, 2012. 
[3] L. F. Hernández-Zimbrón, R. Zamora-Alvarado, L. Ochoa-de la $\mathrm{Paz}$ et al., "Age-related macular degeneration: new paradigms for treatment and management of AMD," Oxidative Medicine and Cellular Longevity, vol. 2018, Article ID 8374647, 14 pages, 2018.

[4] Y. Imamura, S. Noda, K. Hashizume et al., "Drusen, choroidal neovascularization, and retinal pigment epithelium dysfunction in SOD1-deficient mice: a model of age-related macular degeneration," Proceedings of the National Academy of Sciences of the United States of America, vol. 103, no. 30, pp. 11282-11287, 2006.

[5] V. Justilien, J. J. Pang, K. Renganathan et al., "SOD2 knockdown mouse model of early AMD," Investigative Ophthalmology \& Visual Science, vol. 48, no. 10, pp. 4407-4420, 2007.

[6] Z. Zhao, Y. Chen, J. Wang et al., "Age-related retinopathy in NRF2-deficient mice," PLoS One, vol. 6, no. 4, pp. e19456e19456, 2011.

[7] J. C. Khan, D. A. Thurlby, H. Shahid et al., "Smoking and age related macular degeneration: the number of pack years of cigarette smoking is a major determinant of risk for both geographic atrophy and choroidal neovascularisation," The British Journal of Ophthalmology, vol. 90, no. 1, pp. 75-80, 2006.

[8] A. Decanini, C. L. Nordgaard, X. Feng, D. A. Ferrington, and T. W. Olsen, "Changes in select redox proteins of the retinal pigment epithelium in age- related macular degeneration," American Journal of Ophthalmology, vol. 143, no. 4, pp. 607615.e2, 2007.

[9] P. P. Karunadharma, C. L. Nordgaard, T. W. Olsen, and D. A. Ferrington, "Mitochondrial DNA damage as a potential mechanism for age-related macular degeneration," Investigative Ophthalmology \& Visual Science, vol. 51, no. 11, pp. 54705479, 2010.

[10] C. Fang, L. Gu, D. Smerin, S. Mao, and X. Xiong, "The interrelation between reactive oxygen species and autophagy in neurological disorders," Oxidative Medicine and Cellular Longevity, vol. 2017, Article ID 8495160, 16 pages, 2017.

[11] N. Golestaneh, Y. Chu, Y. Y. Xiao, G. L. Stoleru, and A. C. Theos, "Dysfunctional autophagy in RPE, a contributing factor in age-related macular degeneration," Cell Death \& Disease, vol. 8, no. 1, pp. e2537-e2537, 2017.

[12] Y. Zhang, S. D. Cross, J. B. Stanton, A. D. Marmorstein, Y. Z. le, and L. Y. Marmorstein, "Early AMD-like defects in the $\mathrm{RPE}$ and retinal degeneration in aged mice with RPE-specific deletion of Atg5 or Atg7," Molecular Vision, vol. 23, pp. 228241, 2017.

[13] N. A. Abumrad, M. R. el-Maghrabi, E. Z. Amri, E. Lopez, and P. A. Grimaldi, "Cloning of a rat adipocyte membrane protein implicated in binding or transport of long-chain fatty acids that is induced during preadipocyte differentiation. Homology with human CD36.," The Journal of Biological Chemistry, vol. 268, no. 24, pp. 17665-17668, 1993.

[14] R. L. Silverstein and M. Febbraio, "CD36, a scavenger receptor involved in immunity, metabolism, angiogenesis, and behavior," Science Signaling, vol. 2, no. 72, p. re3, 2009.

[15] L. V. Johnson, W. P. Leitner, A. J. Rivest, M. K. Staples, M. J. Radeke, and D. H. Anderson, "The Alzheimer's A beta -peptide is deposited at sites of complement activation in pathologic deposits associated with aging and age-related macular degeneration," Proceedings of the National Academy of Sciences of the United States of America, vol. 99, no. 18, pp. 11830-11835, 2002.
[16] Y. Yamada, J. Tian, Y. Yang et al., "Oxidized low density lipoproteins induce a pathologic response by retinal pigmented epithelial cells," Journal of Neurochemistry, vol. 105, no. 4, pp. 1187-1197, 2008.

[17] K. A. Howes, Y. Liu, J. L. Dunaief et al., "Receptor for advanced glycation end products and age-related macular degeneration," Investigative Ophthalmology \& Visual Science, vol. 45, no. 10, pp. 3713-3720, 2004.

[18] E. Picard, M. Houssier, K. Bujold et al., "CD36 plays an important role in the clearance of oxLDL and associated agedependent sub-retinal deposits," Aging (Albany NY), vol. 2, no. 12, pp. 981-989, 2010.

[19] C. Proulx, É. Picard, D. Boeglin et al., “Azapeptide analogues of the growth hormone releasing peptide 6 as cluster of differentiation 36 receptor ligands with reduced affinity for the growth hormone secretagogue receptor 1a," Journal of Medicinal Chemistry, vol. 55, no. 14, pp. 6502-6511, 2012.

[20] K. Mellal, S. Omri, M. Mulumba et al., "Immunometabolic modulation of retinal inflammation by CD36 ligand," Scientific Reports, vol. 9, no. 1, p. 12903, 2019.

[21] A. Sorsby, "Experimental PIGMENTARY degeneration of the retina by sodium iodate," The British Journal of Ophthalmology, vol. 25, no. 2, pp. 58-62, 1941.

[22] J. Hanus, C. Anderson, D. Sarraf, J. Ma, and S. Wang, "Retinal pigment epithelial cell necroptosis in response to sodium iodate," Cell Death Discovery, vol. 2, no. 1, p. 16054, 2016.

[23] X. Mao, T. Pan, H. Shen, H. Xi, S. Yuan, and Q. Liu, "The rescue effect of mesenchymal stem cell on sodium iodate-induced retinal pigment epithelial cell death through deactivation of NF- $\kappa$ B-mediated NLRP3 inflammasome," Biomedicine \& Pharmacotherapy, vol. 103, pp. 517-523, 2018.

[24] Y.-C. Lin, L. Y. Horng, H. C. Sung, and R. T. Wu, "Sodium iodate disrupted the mitochondrial-lysosomal axis in cultured retinal pigment epithelial cells," Journal of Ocular Pharmacology and Therapeutics, vol. 34, no. 7, pp. 500-511, 2018.

[25] X.-Y. Zhang, T. K. Ng, M. E. Brelén et al., "Continuous exposure to non-lethal doses of sodium iodate induces retinal pigment epithelial cell dysfunction," Scientific Reports, vol. 6, no. 1, article 37279, 2016.

[26] Y. Saito, Y. Kuse, Y. Inoue, S. Nakamura, H. Hara, and M. Shimazawa, "Transient acceleration of autophagic degradation by pharmacological Nrf2 activation is important for retinal pigment epithelium cell survival," Redox Biology, vol. 19, pp. 354-363, 2018.

[27] C.-M. Chan, D. Y. Huang, P. Sekar, S. H. Hsu, and W. W. Lin, "Reactive oxygen species-dependent mitochondrial dynamics and autophagy confer protective effects in retinal pigment epithelial cells against sodium iodate-induced cell death," Journal of Biomedical Science, vol. 26, no. 1, pp. 40-40, 2019.

[28] H. Lin, M. Roh, H. Matsumoto et al., "Blocking the Necroptosis Pathway Decreases RPE and Photoreceptor Damage Induced by NaIO3," bioRxiv, vol. 2018, article 387068, 2018.

[29] R. A. Hazim, S. Volland, A. Yen, B. L. Burgess, and D. S. Williams, "Rapid differentiation of the human RPE cell line, ARPE-19, induced by nicotinamide," Experimental Eye Research, vol. 179, pp. 18-24, 2019.

[30] A. Armento, S. Honisch, V. Panagiotakopoulou et al., "Loss of Complement Factor $\mathrm{H}$ impairs antioxidant capacity and energy metabolism of human RPE cells," bioRxiv, vol. 2020, 2020 . 
[31] A. Baek, S. Yoon, J. Kim et al., “Autophagy and KRT8/keratin 8 protect degeneration of retinal pigment epithelium under oxidative stress," Autophagy, vol. 13, no. 2, pp. 248-263, 2017.

[32] M. Ishiyama, Y. Miyazono, K. Sasamoto, Y. Ohkura, and K. Ueno, "A highly water-soluble disulfonated tetrazolium salt as a chromogenic indicator for NADH as well as cell viability," Talanta, vol. 44, no. 7, pp. 1299-1305, 1997.

[33] C. Wang and R. J. Youle, "The role of mitochondria in apoptosis," Annual Review of Genetics, vol. 43, no. 1, pp. 95-118, 2009.

[34] M. Houssier, W. Raoul, S. Lavalette et al., "CD36 deficiency leads to choroidal involution via COX2 down-regulation in rodents," PLoS Medicine, vol. 5, no. 2, article e39, 2008.

[35] M. Kobayashi and M. Yamamoto, "Nrf2-Keap1 regulation of cellular defense mechanisms against electrophiles and reactive oxygen species," Advances in Enzyme Regulation, vol. 46, no. 1, pp. 113-140, 2006.

[36] A. L. Wang, M. E. Boulton, W. A. Dunn Jr. et al., "Using LC3 to monitor autophagy flux in the retinal pigment epithelium," Autophagy, vol. 5, no. 8, pp. 1190-1193, 2014.

[37] D. J. Klionsky, F. C. Abdalla, H. Abeliovich et al., "Guidelines for the use and interpretation of assays for monitoring autophagy," Autophagy, vol. 8, no. 4, pp. 445-544, 2014.

[38] S. R. Yoshii and N. Mizushima, "Monitoring and measuring autophagy," International Journal of Molecular Sciences, vol. 18, no. 9, p. 1865, 2017.

[39] J. Lee, S. Giordano, and J. Zhang, "Autophagy, mitochondria and oxidative stress: cross-talk and redox signalling," The Biochemical Journal, vol. 441, no. 2, pp. 523-540, 2012.

[40] G. F. Kelso, C. M. Porteous, C. V. Coulter et al., "Selective targeting of a redox-active ubiquinone to mitochondria within cells:", The Journal of Biological Chemistry, vol. 276, no. 7, pp. 4588-4596, 2001.

[41] S. Dikalov, "Cross talk between mitochondria and NADPH oxidases," Free Radical Biology \& Medicine, vol. 51, no. 7, pp. 1289-1301, 2011.

[42] Age-Related Eye Disease Study 2 Research, G, "Lutein + zeaxanthin and omega-3 fatty acids for age-related macular degeneration: the Age-Related Eye Disease Study 2 (AREDS2) randomized clinical trial," JAMA, vol. 309, no. 19, pp. 20052015, 2013.

[43] Age-Related Eye Disease Study Research, G, “A Randomized, placebo-controlled, clinical trial of high-dose supplementation with vitamins $\mathrm{C}$ and $\mathrm{E}$, beta carotene, and zinc for age-related macular degeneration and vision loss: AREDS report No. 8," JAMA Ophthalmology, vol. 119, no. 10, pp. 1417-1436, 2001.

[44] Age-Related Eye Disease Study Research, G, "Risk factors associated with age-related macular degeneration: a case-control study in the age-related eye disease study: age-related eye disease study report number 3," Ophthalmology, vol. 107, no. 12, pp. 2224-2232, 2000.

[45] J. Evans, “Antioxidant supplements to prevent or slow down the progression of AMD: a systematic review and meta-analysis," Eye, vol. 22, no. 6, pp. 751-760, 2008.

[46] C. Proulx, J. Zhang, D. Sabatino, S. Chemtob, H. Ong, and W. D. Lubell, "Synthesis and biomedical potential of Azapeptide modulators of the cluster of differentiation 36 receptor (CD36)," Biomedicine, vol. 8, no. 8, p. 241, 2020.

[47] Sternberg P Jr, P. C. Davidson, D. P. Jones, T. M. Hagen, R. L. Reed, and C. Drews-Botsch, "Protection of retinal pigment epithelium from oxidative injury by glutathione and precur- sors," Investigative Ophthalmology \& Visual Science, vol. 34, no. 13, pp. 3661-3668, 1993.

[48] S. Satish, H. Philipose, R. MAB, and M. Saint-Geniez, "Pharmaceutical induction of PGC- $1 \alpha$ promotes retinal pigment epithelial cell metabolism and protects against oxidative damage," Oxidative Medicine and Cellular Longevity, vol. 2018, Article ID 9248640, 2018.

[49] M. M. Sachdeva, M. Cano, and J. T. Handa, "Nrf2 signaling is impaired in the aging RPE given an oxidative insult," Experimental Eye Research, vol. 119, pp. 111-114, 2014.

[50] S. K. Mitter, C. Song, X. Qi et al., "Dysregulated autophagy in the RPE is associated with increased susceptibility to oxidative stress and AMD," Autophagy, vol. 10, no. 11, pp. 1989-2005, 2014.

[51] E. Itakura, C. Kishi-Itakura, and N. Mizushima, "The hairpintype tail-anchored SNARE syntaxin 17 targets to autophagosomes for fusion with endosomes/lysosomes," Cell, vol. 151, no. 6, pp. 1256-1269, 2012.

[52] L. Sanjurjo, N. Amézaga, G. Aran et al., "The human CD5L/AIM-CD36 axis: a novel autophagy inducer in macrophages that modulates inflammatory responses," Autophagy, vol. 11, no. 3, pp. 487-502, 2015.

[53] J. Li, W. Lin, and L. Zhuang, "CD5L-induced activation of autophagy is associated with hepatoprotection in ischemic reperfusion injury via the CD36/ATG7 axis," Experimental and Therapeutic Medicine, vol. 19, no. 4, pp. 2588-2596, 2020.

[54] A. Iannaccone, T. J. Hollingsworth, D. Koirala et al., "Retinal pigment epithelium and microglia express the CD5 antigenlike protein, a novel autoantigen in age-related macular degeneration," Experimental Eye Research, vol. 155, pp. 64-74, 2017.

[55] A. Uchiyama, J. S. Kim, K. Kon et al., "Translocation of iron from lysosomes into mitochondria is a key event during oxidative stress-induced hepatocellular injury," Hepatology, vol. 48, no. 5, pp. 1644-1654, 2008.

[56] U. T. Brunk and A. Terman, "The mitochondrial-lysosomal axis theory of aging," European Journal of Biochemistry, vol. 269, no. 8, pp. 1996-2002, 2002.

[57] J. Feher, I. Kovacs, M. Artico, C. Cavallotti, A. Papale, and C. Balacco Gabrieli, "Mitochondrial alterations of retinal pigment epithelium in age-related macular degeneration," Neurobiology of Aging, vol. 27, no. 7, pp. 983-993, 2006.

[58] D. A. Ferrington, M. C. Ebeling, R. J. Kapphahn et al., “Altered bioenergetics and enhanced resistance to oxidative stress in human retinal pigment epithelial cells from donors with agerelated macular degeneration," Redox Biology, vol. 13, pp. 255-265, 2017. 